\title{
Spawning habitat selection by the common cuttlefish Sepia officinalis in the Cíes Islands (Northwest Spain)
}

\section{Ángel Guerra ${ }^{\mathrm{a}, *}$}

angelguerra@iim.csic.es

angelguerra@movistar.es

Jorge Hernández-Urcera

Manuel E. Garci

Marta Sestelo

Marcos Regueira

Miguel Gilcoto $^{\mathrm{d}}$

Ángel F. González

a'Instituto de Investigaciones Marinas (CSIC), Department of Natural Resources and Ecology, Eduardo Cabello 6, 36208 Vigo, Spain

bInstituto Español de Oceanografía (IEO), Centro Oceanográfico de Vigo. Subida a Radio Faro 50, 36390 Vigo, Spain

${ }^{\mathbf{c}}$ Centre of Mathematics, University of Minho, Portugal and SiDOR Research Group and CINBIO, University of Vigo, Spain

dInstituto de Investigaciones Marinas (CSIC), Department of Oceanography, Eduardo Cabello 6, 36208 Vigo, Spain

${ }^{*}$ Corresponding author

Handled by George A. Rose

\section{Abstract}

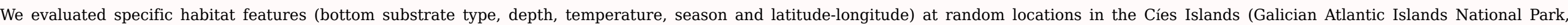

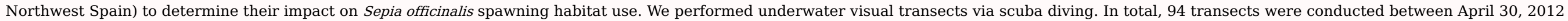

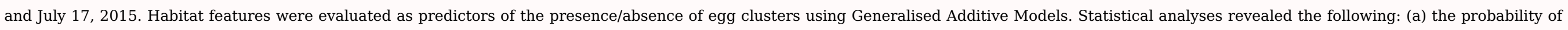

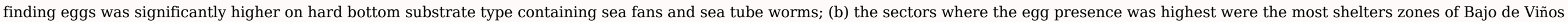

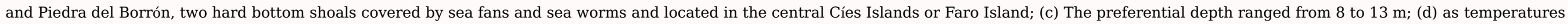

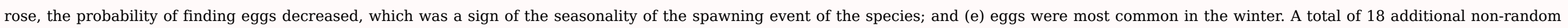

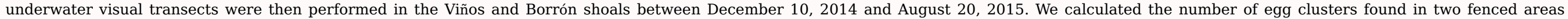

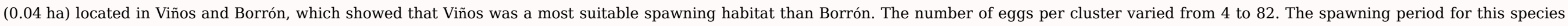

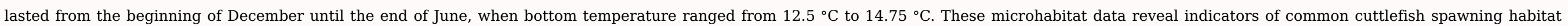
utilisation and should help identify targets for habitat improvement projects and ecosystem management approaches within this national park and elsewhere.

Keywords: Habitat selection; Spawning habitat; Sepia officinalis; NE Atlantic

\section{Introduction}




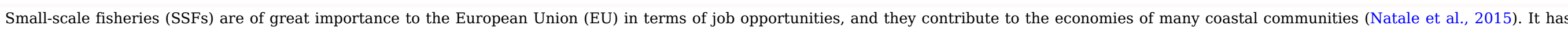

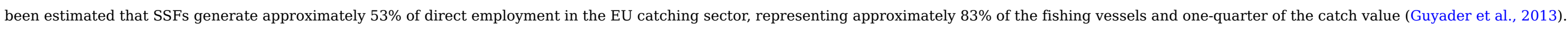

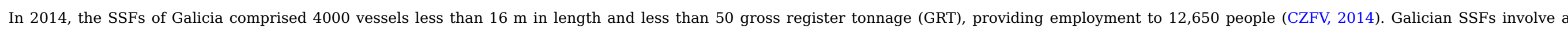

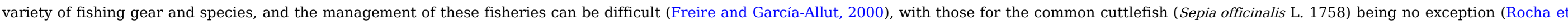
al., 2006).

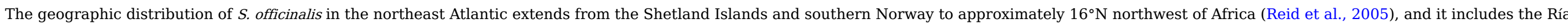

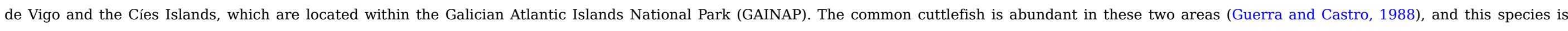
harvested by SSFs using several different types of fishing gear, the most important being trammel nets (Arnaiz et al., 2002).

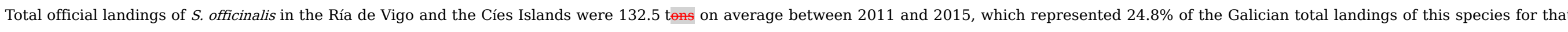

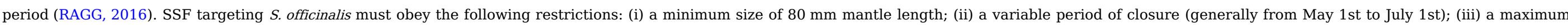
length for the trammel nets; and (iv) fishing must be performed at less than $5 \mathrm{~m}$ depth for boats less than 2.5 GRT (RAGG, 2015).

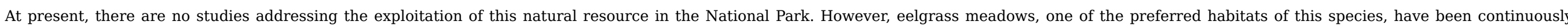

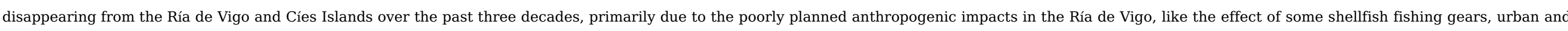

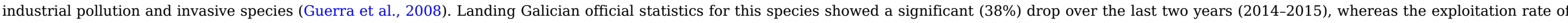

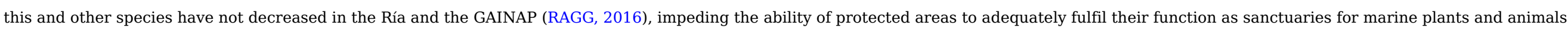

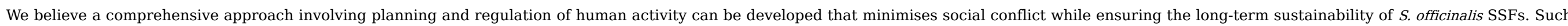

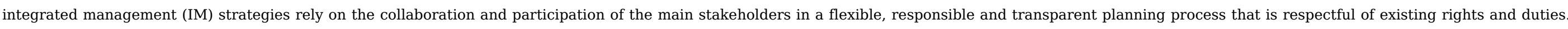

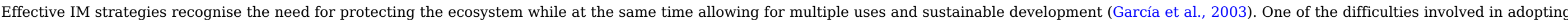

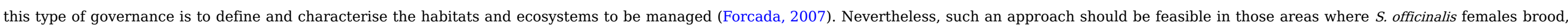
considering that this species commonly spawns in well-defined areas (Bloor et al., 2013).

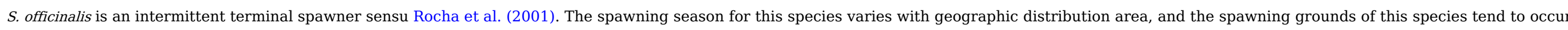

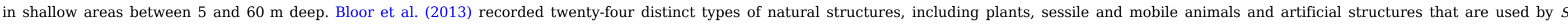
officinalis for egg laying in the northeast Atlantic.

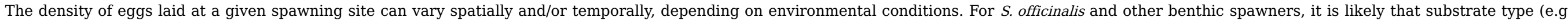

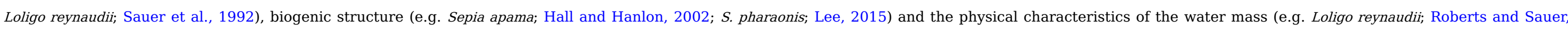
1994) are all instrumental factors in determining the intensity and location of spawning in any given year (Moltschaniwskyj and Pecl, 2003).

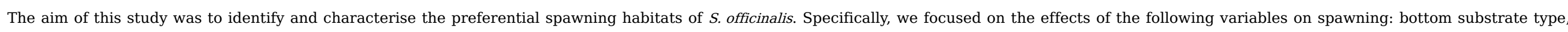

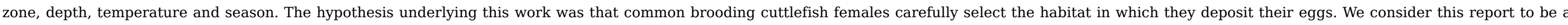

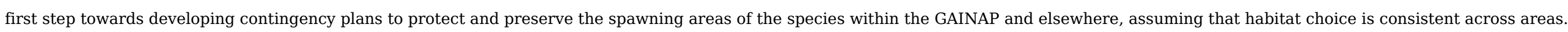

\section{Materials and methods}

\subsection{Study area}

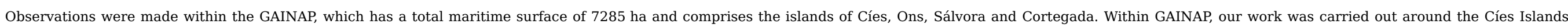
whose maritime area covers 2,637.77 ha and is located at the mouth of the Ría de Vigo (Fig. 1) 


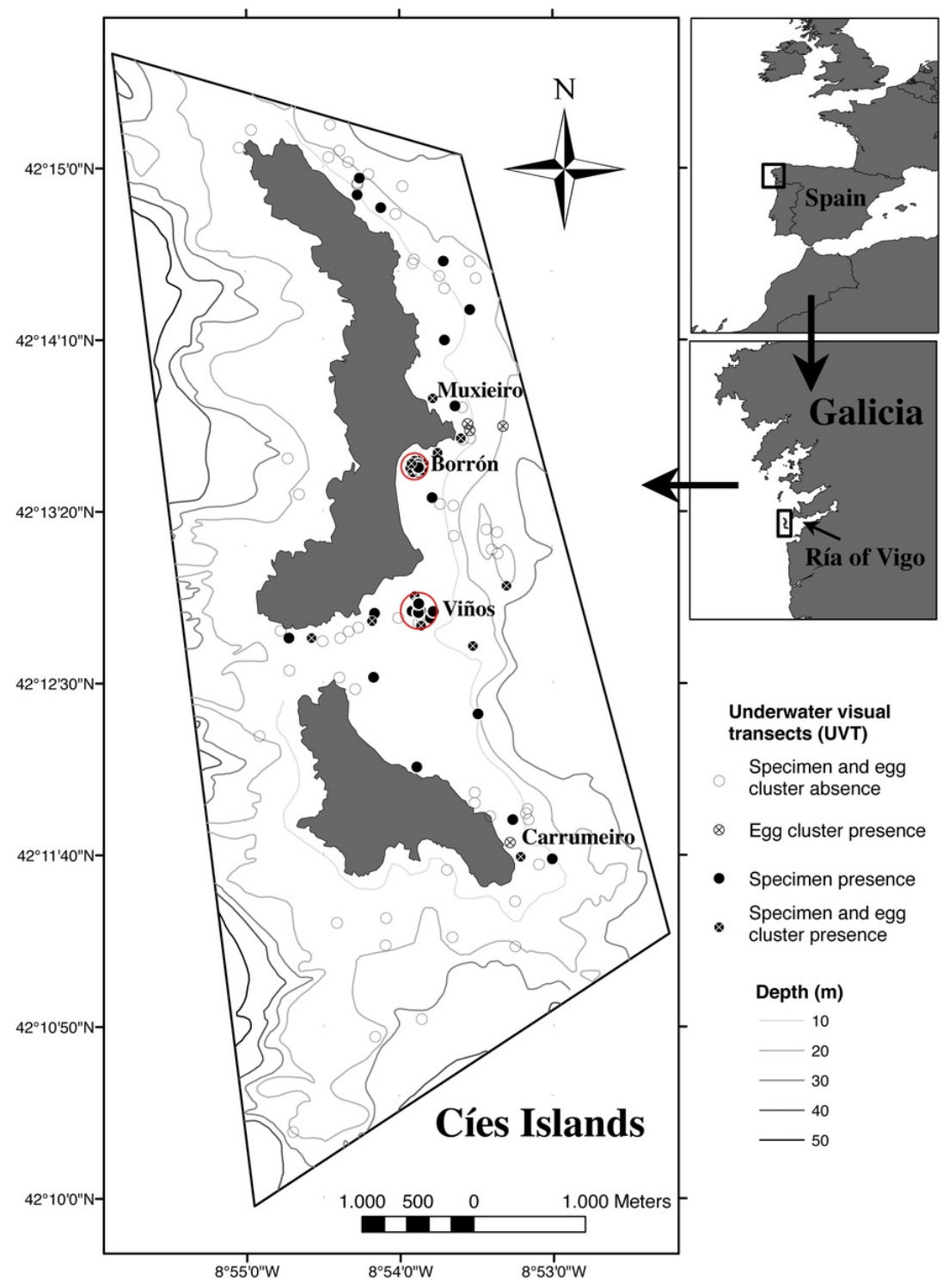

Fig. 1 Mapa de las Islas Cíes mostrando los underwater visual transects (UVT) realizados y la presencia o ausencia de Sepia officinalis egg clusters. alt-text: Fig. 1

\subsection{Underwater visual transects (UVTs)}

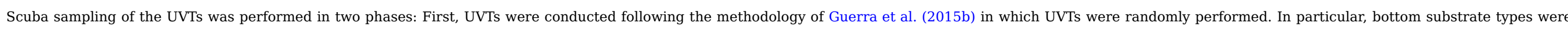

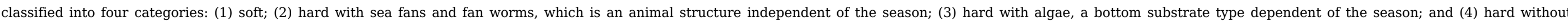

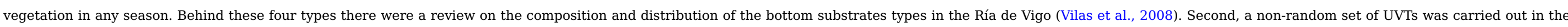

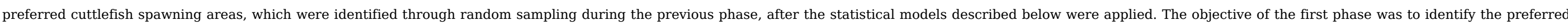




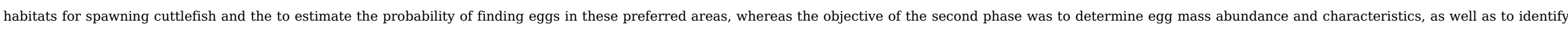
the seasons in which they appeared.

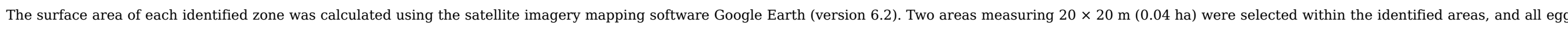
masses within those areas were identified with a numbered buoy

\subsection{Data recording}

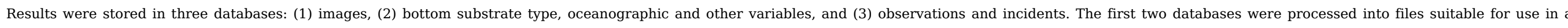

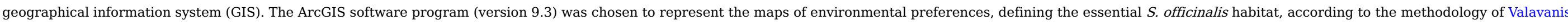
et al. $(2002,2004)$ in the way of the aggregation of abiotic and biotic parameters that are suitable for supporting and sustaining fish population during of stages of the life cycle.

Image analysis was used to determine the number of egg masses in each structure type. The number of eggs in each mass was also estimated from these images.

\subsection{Statistical analysis}

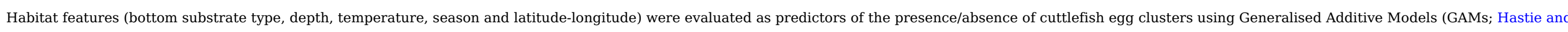
Tibshirani, 1990; Wood, 2006a) on the 94 UVTs performed between April 30, 2012 and July 17, 2014.

A GAM can be expressed as:

$E\left(Y_{i}\right)=g^{-1}\left(X_{i}^{*} \theta^{*}+f_{+1}\left(x_{i 1}\right)+f_{2}\left(x_{i 2}\right)+\ldots+f_{p}\left(x_{i p}\right)\right)$

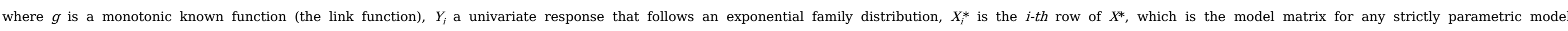
components (such as factors, linear effects, etc.) with corresponding parameter vector $\theta^{*}$, and $f_{1}, f_{2}, \ldots, f_{p}$ are smooth and unknown functions of the covariates $x_{j}(j=1, \ldots, p)$.

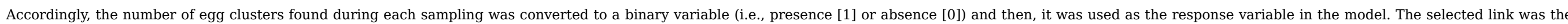
logit function and thin plate regression splines were used as smoothing basis. The optimal degrees of freedom were chosen by means of (Generalised) Cross-Validation (Wood, 2004).

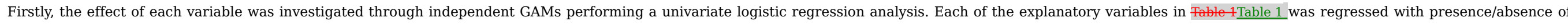

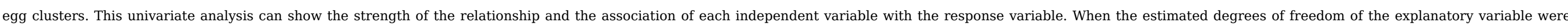

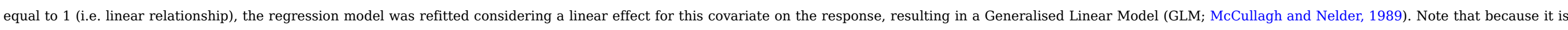
more appropriate to consider the dependence of nests on spatial location as a bivariate surface, latitude and longitude were smoothed as interaction terms using a full tensor product smooth (Wood, 2006b).

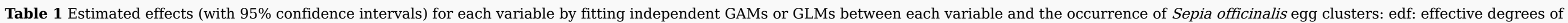
freedom; te (): tensor product; CI: confidence interval; $p$-value: $(* *)<0.05$.

\section{alt-text: Table 1}

\begin{tabular}{|c|c|c|c|}
\hline Effects & edf & Coefficient (95\% CI) & $\mathrm{p}$-value \\
\hline Bottom substrate type 1 & 1.000 & $-3.400(-6.861,-1.686)$ & $0.001 * *$ \\
\hline Bottom substrate type 3 & 1.000 & $-3.099(-5.430,-1.584)$ & $0.001 * *$ \\
\hline Bottom substrate type 4 & 1.000 & $-4.780(-$ Inf, -2.522$)$ & $0.002 * *$ \\
\hline Depth & 1.000 & $-0.156(-0.311,-0.034)$ & $0.024 * *$ \\
\hline Temperature & 1.000 & $-1.056(-1.837,-0.450)$ & $0.003 * *$ \\
\hline Season: Fall & 1.000 & $-2.241(-4.475,-0.737)$ & $0.008 * *$ \\
\hline
\end{tabular}




\begin{tabular}{|c|c|c|c|}
\hline Season: Spring & 1.000 & $-1.653(-3.292,-0.310)$ & 0.022 ** \\
\hline Season: Summer & 1.000 & -3.970 (-Inf, 1.728) & $0.010 * *$ \\
\hline te (Latitude, Longitude) & 3.76 & - & $0.034 * *$ \\
\hline
\end{tabular}

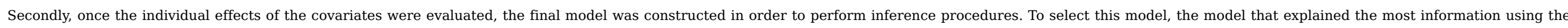

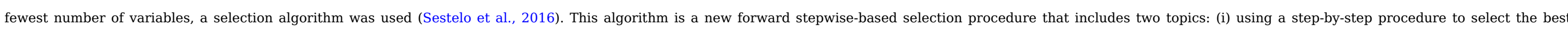
combinations of $q$ variables and (ii) using bootstrap resampling methods to determine the number of covariates to be included in the model.

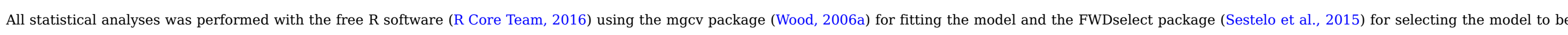
used.

\section{Results}

Data gathered from 94 UVTs, which were randomly performed between April 30, 2012 and July 17, 2015, are shown in the Appendix A. The total swept area in these transects was 126.04 ha.

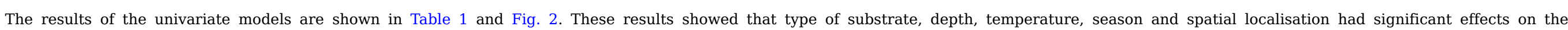
presence/absence of Sepia egg masses. We concluded the following:

i) The probability of finding cuttlefish eggs was greatest in bottom substrate type 2 . The most abundant species of sea fans, tube worms and algae are shown in Table 2 .

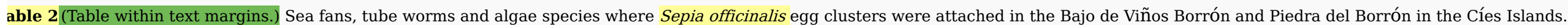

alt-text: Table 2

\begin{tabular}{|c|c|}
\hline Sea fans (Gorgoniidae) & Species \\
\hline & Leptogorgia sarmentosa_(Esper, 1789) \\
\hline & Corynactis viridis (Allman, 1846) \\
\hline & Gymnangium montagui (Billard, 1912) \\
\hline & Eunicella verrucosa (Pallas, 1766) \\
\hline & Nemertesia antennina (Linnaeus, 1758) \\
\hline & Aglaophenia tubulifera (Hincks, 1861) \\
\hline & A. pluma (Linnaeus, 1758) \\
\hline & Calliactis parasítica (Couch, 1842) \\
\hline & Eunicella verrucosa (Pallas, 1766) \\
\hline & Kirchenpaueria pinnata (Linnaeus, 1758) \\
\hline & Alcyonum digitatum (Linnaeus, 1758) \\
\hline Tube worms (Polychaeta, Sabellida) & Sabella spalanzanii (Gmelin, 1791) \\
\hline & Bispira volutacornis (Montagu, 1804) \\
\hline & Serpula vermicularis (Linnaeus, 1758) \\
\hline Algae & Cystoseira spp (mainly baccata, tamariscifolia \\
\hline
\end{tabular}




\section{and usneoides)}

Laminaria ochroleuca

Saccorhiza polyschides

Distyota dichotoma

Dictyopteris polypodioides

Halydris siliquosa

Codium tomentosum

Halimenia cf. latifolia

Scinaia furcellata

ii) The preferred depth for cuttlefish and egg deposits was between 8 and $13 \mathrm{~m}$. Occasionally, eggs were deposited as deep as $25 \mathrm{~m}$.

iii) Increased temperature resulted in a decrease in the response variable. The optimal temperature ranged between $12.5^{\circ} \mathrm{C}$ and $14.75^{\circ} \mathrm{C}$.

iv) The probability of finding cuttlefish eggs was highest in the winter. According to the data from the first sampling phase, the spawning season of $S$. officinalis ranges from December to Apri

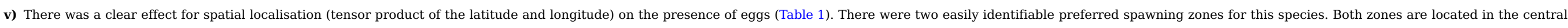
island of Cíes (Faro Island), or more precisely, in the Bajo de Viños and Piedra del Borrón (Fig. 1). 

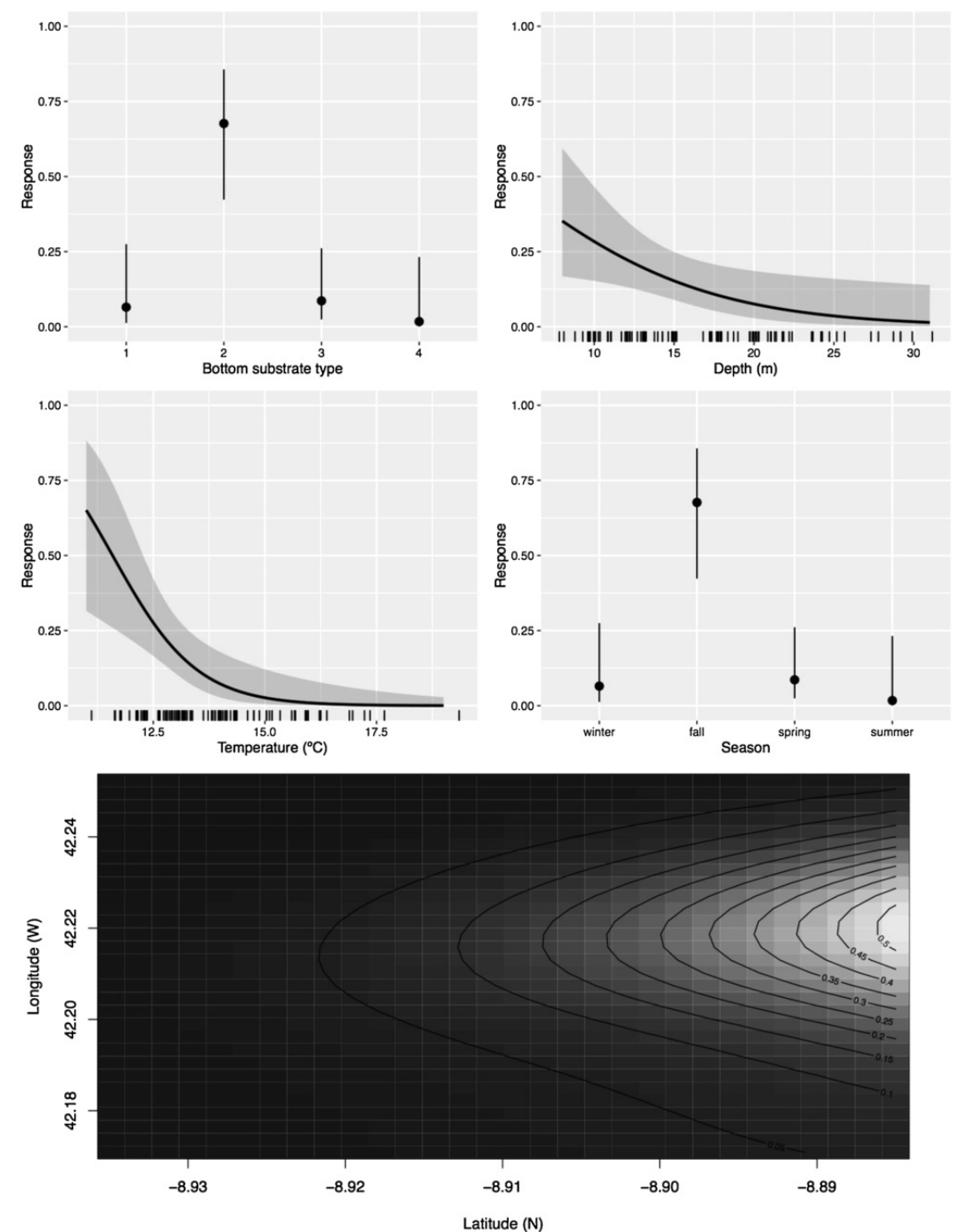

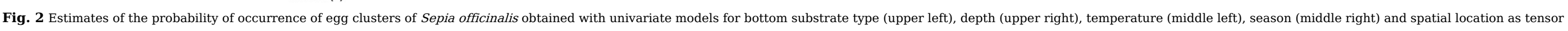
product of latitude and longitude (bottom). The shaded areas and bars indicate the $95 \%$ confidence interval. alt-text: Fig. 2

Table 2 Sea fans, tube worms and algae species where Sepia officinalis (Italics, please.)egg clusters were attached in the Bajo de Viños Borrón and Piedra del Borrón in the Cíes Islands. alt-text: Table 2 
Gymnangium montagui (Billard, 1912)

Eunicella verrucosa (Pallas, 1766)

Nemertesia antennina (Linnaeus, 1758)

Aglaophenia tubulifera (Hincks, 1861)

A. pluma (Linnaeus, 1758)

Calliactis parasítica (Couch, 1842)

Eunicella verrucosa (Pallas, 1766)

Kirchenpaueria pinnata (Linnaeus, 1758)

Alcyonum digitatum (Linnaeus, 1758)

Tube worms (Polychaeta, Sabellida)

Sabella spalanzanii (Gmelin, 1791)

Bispira volutacornis (Montagu, 1804)

Serpula vermicularis (Linnaeus, 1758)

Algae

Cystoseira spp (mainly baccata, tamariscifolia and usneoides)

\section{Laminaria ochroleuca}

Saccorhiza polyschides

Distyota dichotoma,

Dictyopteris polypodioides

Halydris siliquosa

Codium tomentosum

Halimenia cf. latifolia

Scinaia furcellata

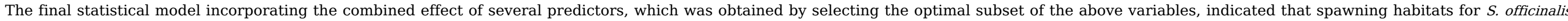

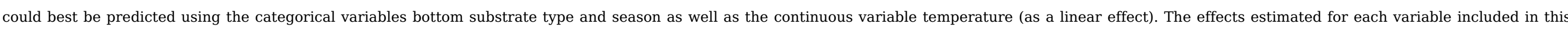
model (together with their 95\% confidence intervals) are shown in Table 3. Fig. 3 shows the partial effects of each of these variables.

Table 3 Estimated effects (with 95\% confidence intervals) for each variable for each variable included in the GLM final model. CI: confidence interval; p-value: $(* *)<0.05$, $(*)<0.1$.

alt-text: Table 3

\begin{tabular}{|c|c|c|c|}
\hline Effects & edf & Coefficient (95\% CI) & $\mathrm{p}$-value \\
\hline Bottom substrate type 1 & 1.000 & $-3.200(-9.001,-0.839)$ & 0.016 ** \\
\hline Bottom substrate type 3 & 1.000 & $-2.524(-6.923,-0.546)$ & 0.020 ** \\
\hline Bottom substrate type 4 & 1.000 & $-5.356(-\operatorname{Inf},-2.769)$ & $0.001 * *$ \\
\hline Temperature & 1.000 & $-0.786(-2.743,-0.097)$ & $0.042 * *$ \\
\hline
\end{tabular}




\begin{tabular}{|c|c|c|c|}
\hline Season: Fall & 1.000 & $-1.345(-5.979,2.048)$ & 0.325 \\
\hline Season: Spring & 1.000 & $0.221(-2.297,4.114)$ & 0.854 \\
\hline Season: Summer & 1.000 & -3.273 (-Inf, 0.387) & 0.067 \\
\hline
\end{tabular}
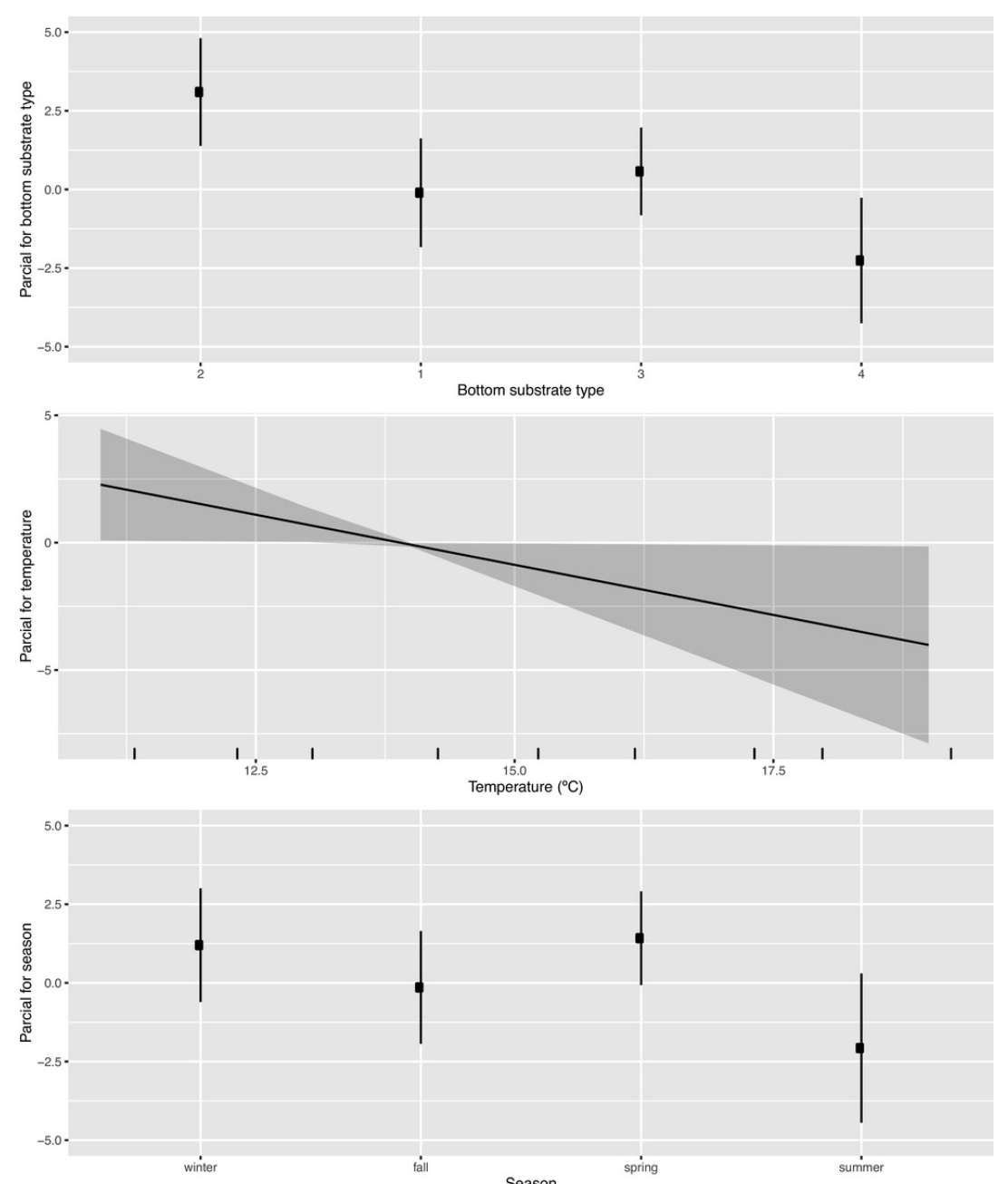

Fig. 3 Parcial effects of the covariates on the occurrence of egg clusters of Sepia officinalis obtained with the final model. The shaded area and the bars indicate the $95 \%$ confidence interval for the partial effects. alt-text: Fig. 3

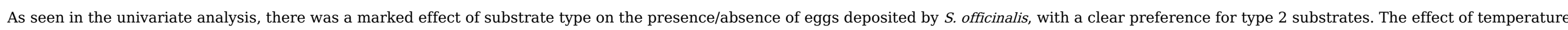

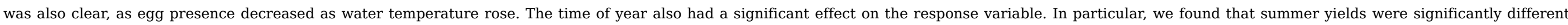
(negative) from yields during colder seasons.

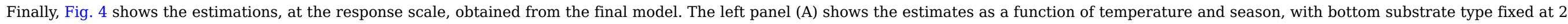



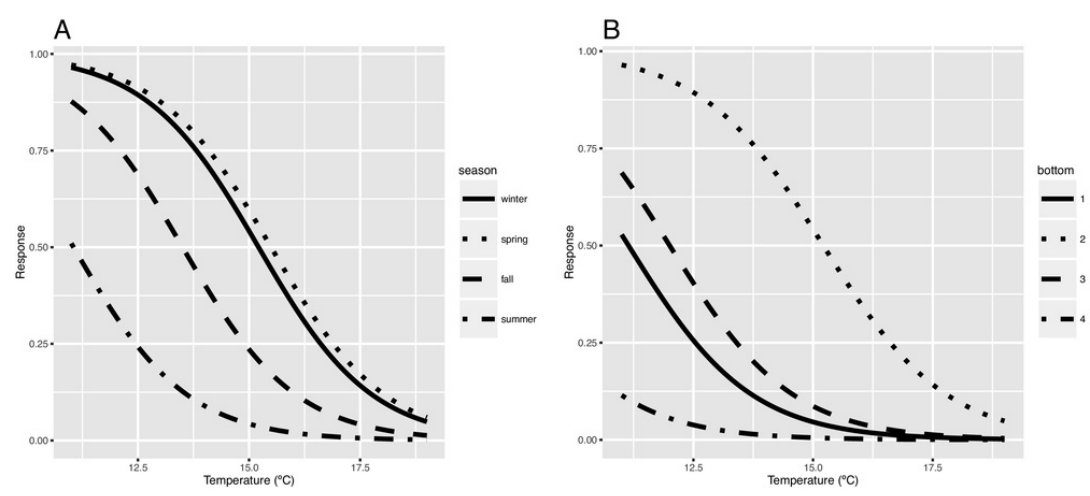

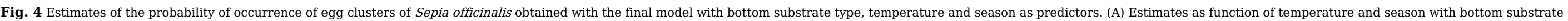
type fixed a 2; (B) estimates as a function of the temperature and bottom substrate type with the season fixed as winter.

\section{alt-text: Fig. 4}

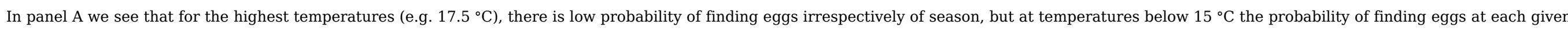

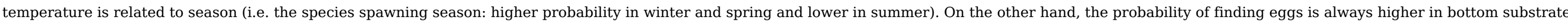

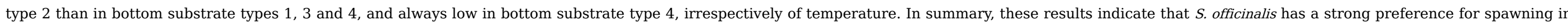
zones with bottom substrate type 2 in spring and winter, and within these seasons in zones where bottom temperature is below $15{ }^{\circ} \mathrm{C}$.

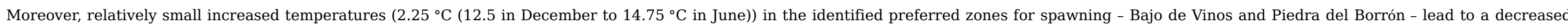

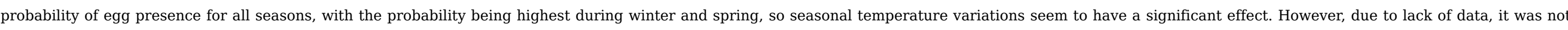
possible to determine which season had the highest probability ( $p$ value $=0.854$ ).

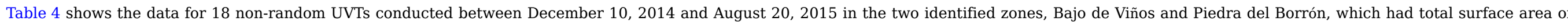

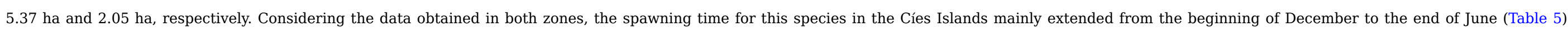
Bottom temperature ranged between $12.5^{\circ} \mathrm{C}$ and $14.75^{\circ} \mathrm{C}$. Courtship, copulation, spawning and hatching were observed in both zones (Viños and Borrón).

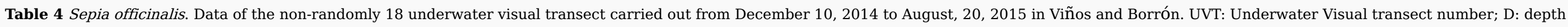

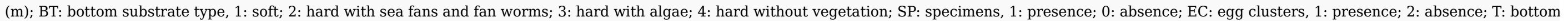
temperature ( ${ }^{\circ}$ Celsius); Ila: initial latitude (N); Ilo: initial longitude (W).

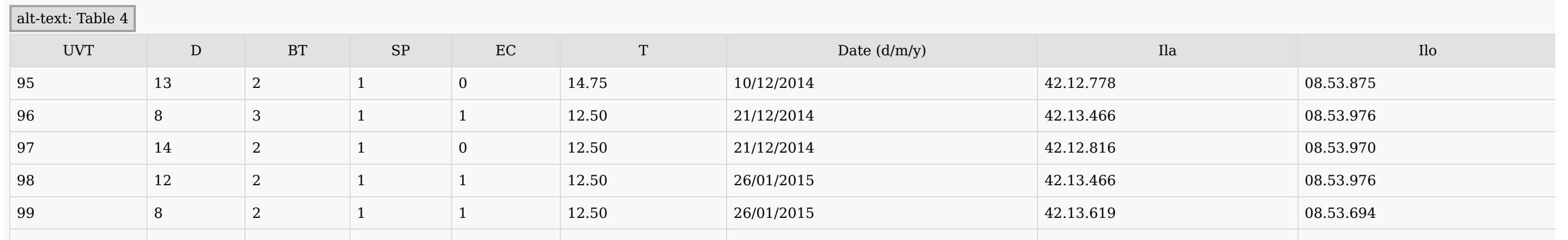




\begin{tabular}{|c|c|c|c|c|c|c|c|c|}
\hline 100 & 12 & 2 & 1 & 1 & 12.65 & $10 / 02 / 2015$ & 42.13 .812 & 08.53 .877 \\
\hline 101 & 8 & 2 & 1 & 1 & 12.50 & $10 / 02 / 2015$ & 42.13 .472 & 08.53.977 \\
\hline 102 & 7 & 2 & 1 & 1 & 12.65 & $12 / 03 / 2015$ & 42.13 .489 & 08.54 .000 \\
\hline 103 & 8 & 2 & 1 & 1 & 12.65 & $12 / 03 / 2015$ & 42.13 .472 & 08.53 .977 \\
\hline 104 & 8 & 2 & 1 & 1 & 13.65 & 08/04/2015 & 42.13 .477 & 08.54 .016 \\
\hline 105 & 12 & 2 & 1 & 1 & 13.60 & 08/04/2015 & 42.12 .709 & 08.53 .954 \\
\hline 106 & 24 & 1 & 0 & 0 & 13.35 & $16 / 04 / 2015$ & 42.14 .487 & 08.54 .000 \\
\hline 107 & 11 & 2 & 0 & 0 & 13.60 & $16 / 04 / 2015$ & 42.13 .768 & 08.53 .686 \\
\hline 108 & 8 & 2 & 1 & 1 & 13.45 & $25 / 04 / 2015$ & 42.13 .477 & 08.54 .016 \\
\hline 109 & 19 & 4 & 0 & 0 & 14.50 & $25 / 05 / 2015$ & 42.13 .347 & 08.54 .750 \\
\hline 110 & 8 & 3 & 1 & 1 & 13.65 & $25 / 05 / 2015$ & 42.13 .477 & 08.54 .016 \\
\hline 110 & 12 & 3 & 1 & 1 & 14.35 & $11 / 06 / 2015$ & 42.13 .466 & 08.53 .976 \\
\hline 111 & 8 & 3 & 1 & 0 & 14.75 & $11 / 06 / 2015$ & 42.13 .477 & 08.54 .016 \\
\hline 112 & 8 & 1 & 1 & 0 & 14.75 & $28 / 07 / 2015$ & 42.13 .477 & 08.54 .016 \\
\hline 113 & 9 & 3 & 1 & 1 & 14.75 & $20 / 08 / 2015$ & 42.13 .477 & 08.54 .016 \\
\hline
\end{tabular}

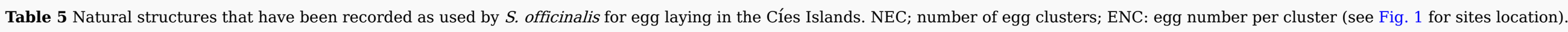
alt-text: Table 5

\begin{tabular}{|c|c|c|c|c|}
\hline VCN & Date $(\mathrm{d} / \mathrm{m} / \mathrm{y})$ & Site & Structure & NEC (ENC) \\
\hline 1 & $30 / 03 / 2012$ & Bajo de Viños & Inside an old creel & 1 \\
\hline 38 & $17 / 01 / 2013$ & Bajo de Viños & Leptogorgia sarmentosa + Sabella spallanzani & 2 \\
\hline 41 & $04 / 02 / 2013$ & Cantareira, North of Muxieiro & Leptogorgia sarmentosa + Sabella spallanzani & 2 \\
\hline 43 & $28 / 02 / 2013$ & Bajo de Viños & Leptogorgia sarmentosa & 4 \\
\hline 44 & $27 / 03 / 2013$ & Muxieiro & Leptogorgia sarmentosa & 2 \\
\hline 78 & $05 / 12 / 2013$ & Los Ciegos-West of the Borrón & Leptogorgia sarmentosa & 2 \\
\hline 79 & $05 / 12 / 2013$ & Freu da Porta- West of Viños & Leptogorgia sarmentosa & 1 \\
\hline 82 & $19 / 02 / 2014$ & Muxieiro & Leptogorgia sarmentosa & 1 \\
\hline 83 & $19 / 02 / 2014$ & Piedra del Borrón & Sabella spallanzani & 2 \\
\hline 84 & $07 / 03 / 2014$ & Carrumeiro & Leptogorgia sarmentosa & 1 \\
\hline 86 & $12 / 03 / 2014$ & Xesteira, South of Carrumeiro & Leptogorgia sarmentosa & 1 \\
\hline 88 & $20 / 03 / 2014$ & Freu da Porta- West of Viños & Leptogorgia sarmentosa & 1 \\
\hline 90 & $10 / 04 / 2014$ & Cantareira North of Muxieiro & Leptogorgia sarmentosa & 1 \\
\hline
\end{tabular}




\begin{tabular}{|c|c|c|c|c|}
\hline 96 & $21 / 12 / 2014$ & Piedra del Borrón & Cystoseira spp. & 1 \\
\hline 98 & $26 / 01 / 2015$ & Bajo de Viños & Leptogorgia sarmentosa & 1 \\
\hline 99 & $26 / 01 / 2015$ & Piedra del Borrón & Leptogorgia sarmentosa & 1 \\
\hline 100 & $10 / 02 / 2015$ & Bajo de Viños & Leptogorgia sarmentosa & $1(12)$ \\
\hline \multirow[t]{6}{*}{101} & $10 / 02 / 2015$ & Bajo de Viños & Leptogorgia sarmentosa & $1(11)$ \\
\hline & & Bajo de Viños & Leptogorgia sarmentosa & $1(60)$ \\
\hline & & Bajo de Viños & Leptogorgia sarmentosa & $4(18,20,9,55)$ \\
\hline & & Bajo de Viños & Leptogorgia sarmentosa & $1(45)$ \\
\hline & & Bajo de Viños & Leptogorgia sarmentosa & $1(40)$ \\
\hline & & Bajo de Viños & Leptogorgia sarmentosa & $2(20,20)$ \\
\hline \multirow[t]{21}{*}{$102 / 103$} & $12 / 03 / 2015$ & Bajo de Viños & Halydris silicuosa & $1(8)$ \\
\hline & & Bajo de Viños & Cystoseira spp. & $1(82)$ \\
\hline & & Bajo de Viños & Cystoseira spp. & $1(60)$ \\
\hline & & Bajo de Viños & Cystoseira spp. & $1(20)$ \\
\hline & & Bajo de Viños & Cystoseira spp. & $1(40)$ \\
\hline & & Bajo de Viños & Leptogorgia sarmentosa & $1(6)$ \\
\hline & & Bajo de Viños & Leptogorgia sarmentosa & $1(15)$ \\
\hline & & Bajo de Viños & Leptogorgia sarmentosa & $1(20)$ \\
\hline & & Bajo de Viños & Leptogorgia sarmentosa & $1(4)$ \\
\hline & & Bajo de Viños & Leptogorgia sarmentosa & $1(10)$ \\
\hline & & Bajo de Viños & Leptogorgia sarmentosa & $1(12)$ \\
\hline & $12 / 03 / 2015$ & Bajo de Viños & Leptogorgia sarmentosa & $5(20,40,60,60,20)$ \\
\hline & & Bajo de Viños & Leptogorgonia sarmentosa & $2(25,15)$ \\
\hline & & Bajo de Viños & Cystoseira spp. & $2(25,12)$ \\
\hline & & Bajo de Viños & Cystoseira spp. & $3(7,46,20)$ \\
\hline & & Bajo de Viños & Cystoseira spp. & $1(30)$ \\
\hline & & Bajo de Viños & Cystoseira spp. & $2(60,10)$ \\
\hline & & Bajo de Viños & Cystoseira spp. & $2(30,14)$ \\
\hline & & Bajo de Viños & Cystoseira spp. & $1(13)$ \\
\hline & & Bajo de Viños & Cystoseira spp. & $1(45)$ \\
\hline & & Bajo de Viños & Sabella spallanzani & $1(74)$ \\
\hline 104 & 08/04/2015 & Piedra del Borrón & Cystoseira spp. & $1(25)$ \\
\hline
\end{tabular}




\begin{tabular}{|c|c|c|c|c|}
\hline & & Piedra del Borrón & Cystoseira spp. & $3(2,30,15)$ \\
\hline & & Piedra del Borrón & Cystoseira spp. & $1(40)$ \\
\hline & & Piedra del Borrón & Cystoseira spp. & $2(20,15)$ \\
\hline & & Piedra del Borrón & Cystoseira spp. & $2(30,15)$ \\
\hline & & Piedra del Borrón & Cystoseira spp. & $2(60,20)$ \\
\hline & & Piedra del Borrón & Cystoseira spp. & $1(40)$ \\
\hline & & Piedra del Borrón & Halimenia cf. latifolia & $3(40,45,26)$ \\
\hline \multirow[t]{4}{*}{105} & $08 / 04 / 2015$ & Piedra del Borrón & Leptogorgia sarmentosa & $1(30)$ \\
\hline & & Bajo de Viños & Leptogorgia sarmentosa & $2(14)$ \\
\hline & & Bajo de Viños & Leptogorgia sarmentosa & $4(12,15,20,20)$ \\
\hline & & Bajo de Viños & Leptogorgia sarmentosa & $4(10,8,15,20)$ \\
\hline 108 & $25 / 04 / 2015$ & Piedra del Borrón & Leptogorgia sarmentosa & $1(6)$ \\
\hline 110 & $25 / 05 / 2015$ & Piedra del Borrón & Cabo del boyarín & 1 \\
\hline 111 & $11 / 06 / 2015$ & Bajo de Viños & Leptogorgia sarmentosa & 2 \\
\hline 112 & $11 / 06 / 2015$ & Piedra del Borrón & Leptogorgia sarmentosa & 2 \\
\hline 113 & $28 / 07 / 2015$ & Piedra del Borrón & Leptogorgia sarmentosa & 2 \\
\hline
\end{tabular}

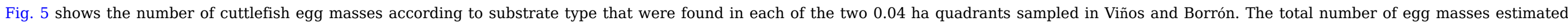

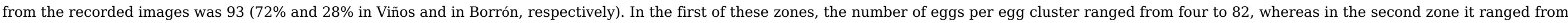
six to 74 (Table 5).

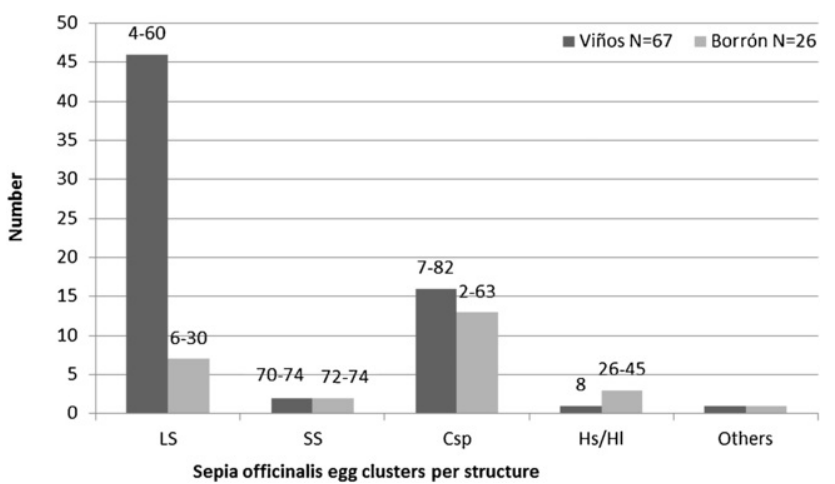

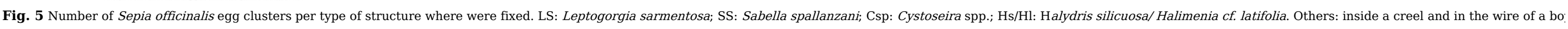
The minimum and maximum number of eggs per type of structure indicated on the top of each histogram, in "others" this data is unknown.

alt-text: Fig. 5

\section{Discussion}




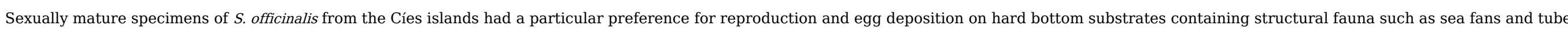

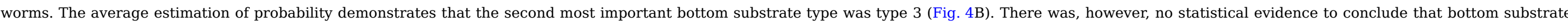

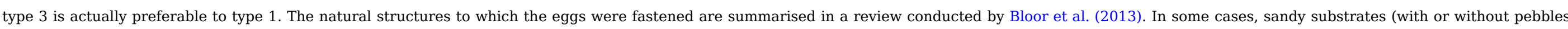
and/or rocks) and associated flora (e.g., seagrass or seaweeds), as well as artificial substrata, were also utilised.

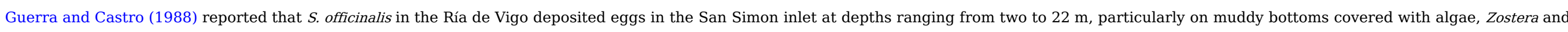

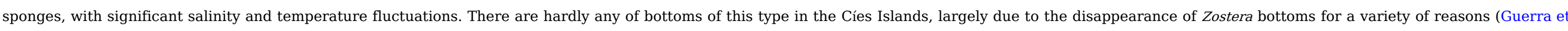

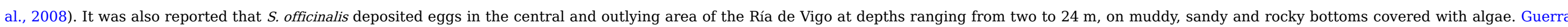

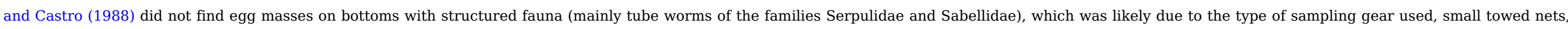
that were not employed on this bottom substrate type to avoid habitat destruction.

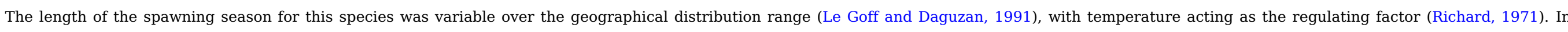

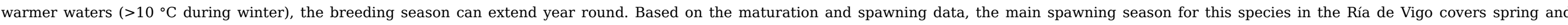

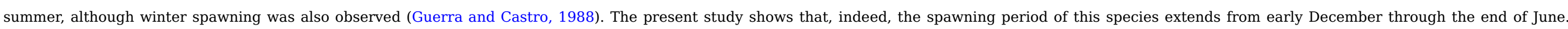

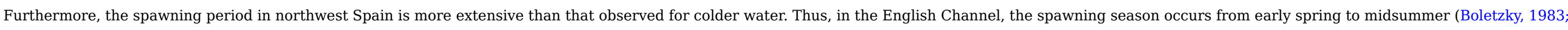
Le Goff and Daguzan, 1991; Dunn, 1999).

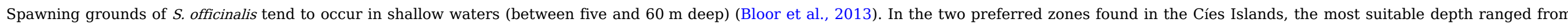
eight to $13 \mathrm{~m}$. However, one egg cluster was found at a depth of $25 \mathrm{~m}$.

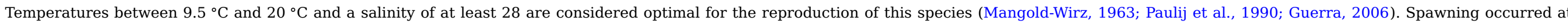

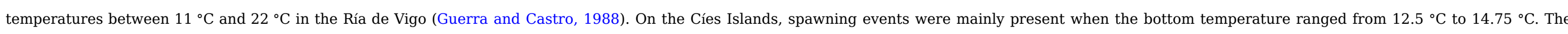

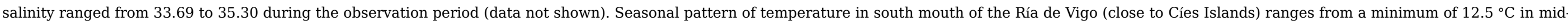

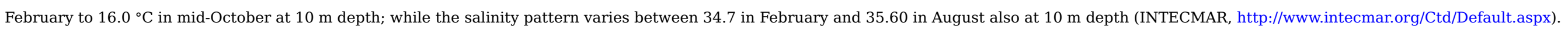

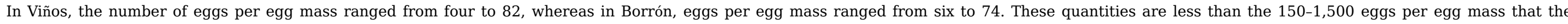

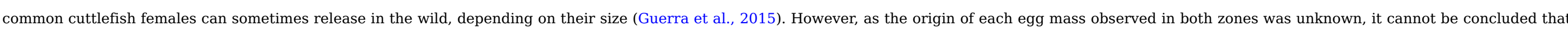

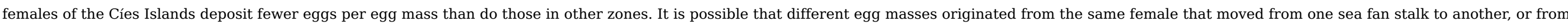

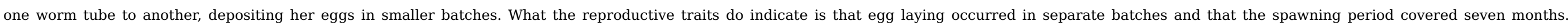

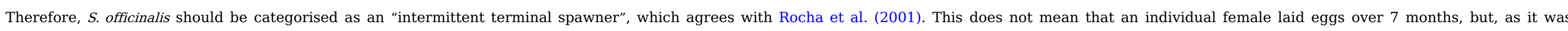
observed, that females do not lay all her eggs simultaneously, but in several batches and during a relatively extensive period.

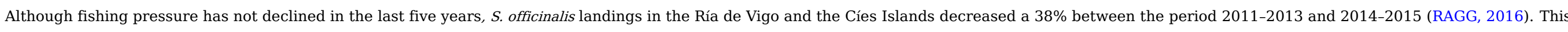

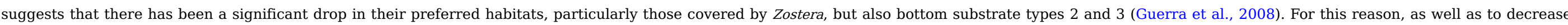

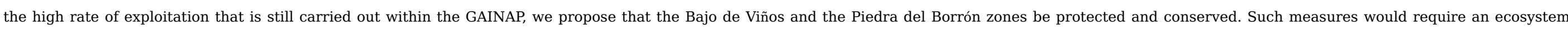

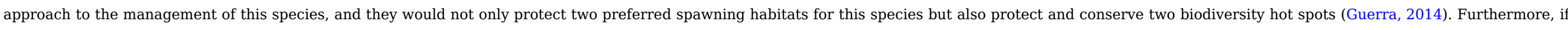

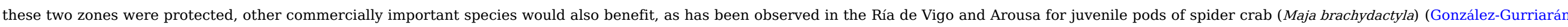

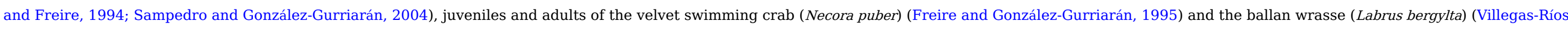
et al., 2013).

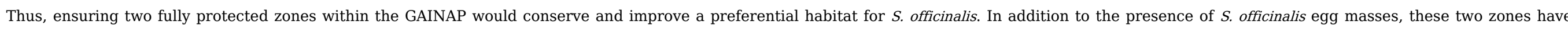

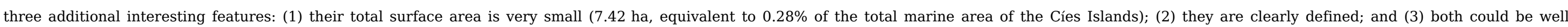

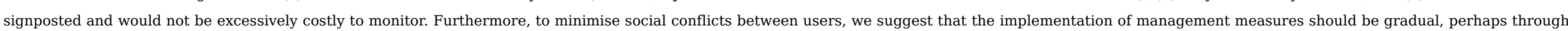




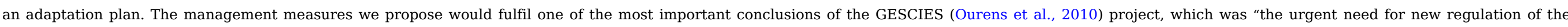

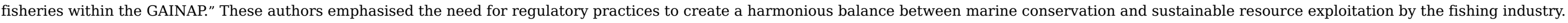

\section{Acknowledgements}

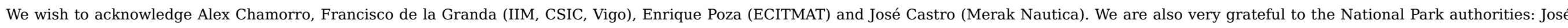

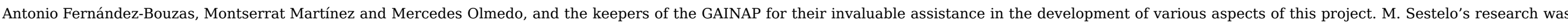

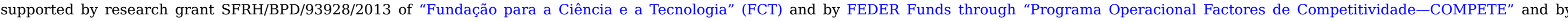

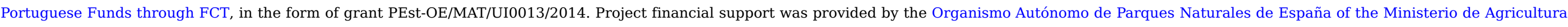
Alimentación y Medio Ambiente (CEFAPARQUES, Project number: 458/2011).

\section{Appendix A}

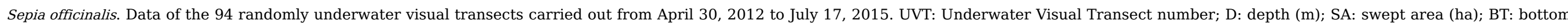

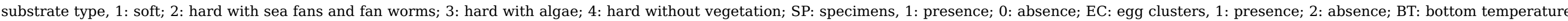
( ${ }^{\circ}$ Celsius); Ila: latitude initial (N); Ilo: longitude initial (W).

\begin{tabular}{|c|c|c|c|c|c|c|c|c|c|}
\hline UVT & D & SA & $\mathrm{BT}$ & SP & $\mathrm{EC}$ & BT & Date $(\mathrm{d} / \mathrm{m} / \mathrm{y})$ & Ila & Ilo \\
\hline 1 & 13 & 1.128 & 1 & 1 & 1 & 14 & $30 / 03 / 2012$ & 4212.768 & 0853.981 \\
\hline 2 & 15 & 3.720 & 1 & 1 & 0 & 13 & $17 / 04 / 2012$ & 4211.767 & 0853.357 \\
\hline 3 & 13 & 2.580 & 3 & 1 & 0 & 13 & $17 / 04 / 2012$ & 4212.746 & 0853.894 \\
\hline 4 & 13 & 1.664 & 3 & 0 & 1 & 13 & 07/05/2012 & 4213.466 & 0853.976 \\
\hline 5 & 29 & 0.140 & 4 & 0 & 0 & 14 & $14 / 05 / 2012$ & 4213.521 & 0854.824 \\
\hline 6 & 15 & 1.024 & 1 & 1 & 0 & 14 & $14 / 06 / 2012$ & 4213.330 & 0853.882 \\
\hline 7 & 18 & 0.405 & 3 & 1 & 0 & 15 & $23 / 05 / 2012$ & 4211.577 & 0853.100 \\
\hline 8 & 9 & 0.441 & 1 & 1 & 0 & 16 & $23 / 05 / 2012$ & 4212.769 & 0854.258 \\
\hline 9 & 21 & 1.000 & 3 & 0 & 0 & 16 & $04 / 06 / 2012$ & 4210.715 & 0854.257 \\
\hline 10 & 20 & 1.280 & 2 & 1 & 0 & 17 & $04 / 06 / 2012$ & 4212.650 & 0854.816 \\
\hline 11 & 17 & 2.160 & 3 & 0 & 0 & 16 & 20/06/2012 & 4215.115 & 0855.061 \\
\hline 12 & 20 & 1.120 & 3 & 0 & 0 & 16 & $20 / 06 / 2012$ & 4214.848 & 0854.367 \\
\hline 13 & 19 & 1.760 & 3 & 0 & 0 & 16 & 09/07/2012 & 4214.467 & 0854.008 \\
\hline 14 & 20 & 0.630 & 3 & 1 & 0 & 16 & 09/07/2012 & 4214.842 & 0854.369 \\
\hline 15 & 20 & 1.720 & 3 & 0 & 0 & 15 & $17 / 07 / 2012$ & 4214.858 & 0854.367 \\
\hline 16 & 13 & 1.696 & 3 & 0 & 0 & 16 & $17 / 07 / 2012$ & 4212.681 & 0854.428 \\
\hline 17 & 15 & 0.425 & 3 & 0 & 0 & 15 & $24 / 07 / 2012$ & 4211.820 & 0853.261 \\
\hline 18 & 17 & 0.560 & 3 & 0 & 0 & 13 & $27 / 07 / 2012$ & 4214.406 & 0853.834 \\
\hline 19 & 26 & 0.336 & 1 & 1 & 0 & 13 & $27 / 07 / 2012$ & 4212.281 & 0853.585 \\
\hline
\end{tabular}




\begin{tabular}{|c|c|c|c|c|c|c|c|c|c|}
\hline 20 & 10 & 0.096 & 1 & 1 & 0 & 13 & 27/07/2012 & 4212.459 & 0854.265 \\
\hline 21 & 27 & 0.036 & 3 & 0 & 0 & 13 & 08/08/2012 & 4212.401 & 0854.385 \\
\hline 22 & 10 & 1.092 & 3 & 0 & 0 & 14 & 08/08/2012 & 4212.458 & 0854.488 \\
\hline 23 & 30 & 2.240 & 4 & 0 & 0 & 14 & 27/08/2012 & 4215.015 & 0854.485 \\
\hline 24 & 18 & 2.220 & 3 & 0 & 0 & 14 & $27 / 08 / 2012$ & 4214.983 & 0854.556 \\
\hline 25 & 29 & 0.096 & 3 & 0 & 0 & 14 & 05/09/2012 & 4210.253 & 0854.793 \\
\hline 26 & 15 & 1.040 & 3 & 0 & 0 & 14 & 05/09/2012 & 4210.428 & 0853.953 \\
\hline 27 & 18 & 0.015 & 1 & 0 & 0 & 14 & $21 / 09 / 2012$ & 4211.767 & 0853.255 \\
\hline 28 & 10 & 0.648 & 3 & 0 & 0 & 14 & 21/09/2012 & 4211.942 & 0853.607 \\
\hline 29 & 10 & 0.516 & 3 & 0 & 0 & 14 & $21 / 09 / 2012$ & 4211.848 & 0853.607 \\
\hline 30 & 19 & 1.280 & 1 & 1 & 0 & 15 & 10/10/2012 & 4214.243 & 0853.636 \\
\hline 31 & 24 & 0.600 & 3 & 1 & 0 & 15 & 10/10/2012 & 4214.881 & 0854.354 \\
\hline 32 & 17 & 0.768 & 5 & 0 & 0 & 17 & 29/10/2012 & 4214.708 & 0854.118 \\
\hline 33 & 15 & 1.560 & 3 & 1 & 0 & 17 & 29/10/2012 & 4213.775 & 0853.732 \\
\hline 34 & 20 & 1.080 & 3 & 0 & 0 & 16 & 09/11/2012 & 4211.290 & 0854.186 \\
\hline 35 & 18 & 1.520 & 5 & 0 & 0 & 16 & 09/11/2012 & 4211.152 & 0853.343 \\
\hline 36 & 18 & 1.024 & 5 & 0 & 0 & 12 & $11 / 12 / 2012$ & 4214.346 & 0853.801 \\
\hline 37 & 15 & 1.568 & 1 & 0 & 0 & 13 & $11 / 12 / 2012$ & 4212.747 & 0854.104 \\
\hline 38 & 13 & 1.600 & 2 & 0 & 1 & 13 & $17 / 01 / 2013$ & 4212.782 & 0853.960 \\
\hline 39 & 12 & 1.536 & 3 & 1 & 0 & 13 & $17 / 01 / 2013$ & 4214.095 & 0853.801 \\
\hline 40 & 21 & 1.032 & 5 & 0 & 0 & 13 & 04/02/2013 & 4214.857 & 0854.368 \\
\hline 41 & 12 & 1.752 & 2 & 1 & 1 & 13 & $04 / 02 / 2013$ & 4212.852 & 0853.996 \\
\hline 42 & 24 & 0.420 & 5 & 0 & 0 & 13 & $28 / 02 / 2013$ & 4211.197 & 0853.754 \\
\hline 43 & 12 & 1.856 & 2 & 1 & 1 & 12 & 28/02/2013 & 4212.610 & 0853.617 \\
\hline 44 & 13 & 0.402 & 2 & 0 & 1 & 13 & $27 / 03 / 2013$ & 4213.656 & 0853.635 \\
\hline 45 & 17 & 1.710 & 5 & 0 & 0 & 14 & $19 / 04 / 2013$ & 4212.635 & 0854.597 \\
\hline 46 & 12 & 1.248 & 1 & 0 & 0 & 14 & $19 / 04 / 2013$ & 4212.752 & 0853.957 \\
\hline 47 & 25 & 0.180 & 5 & 0 & 0 & 13 & $30 / 04 / 2013$ & 4212.174 & 0855.010 \\
\hline 48 & 9 & 0.756 & 1 & 1 & 0 & 13 & $30 / 04 / 2013$ & 4212.024 & 0853.984 \\
\hline 49 & 22 & 0.758 & 5 & 0 & 0 & 12 & $24 / 05 / 2013$ & 4211.159 & 0854.189 \\
\hline 50 & 10 & 1.480 & 1 & 1 & 0 & 12 & $24 / 05 / 2013$ & 4212.780 & 0854.013 \\
\hline 51 & 24 & 0.732 & 5 & 0 & 0 & 12 & $28 / 05 / 2013$ & 4214.848 & 0854.367 \\
\hline
\end{tabular}




\begin{tabular}{|c|c|c|c|c|c|c|c|c|c|}
\hline 52 & 10 & 1.575 & 5 & 0 & 0 & 12 & 04/06/2013 & 4213.618 & 0853.632 \\
\hline 53 & 12 & 9.360 & 2 & 0 & 0 & 14 & 04/06/2013 & 4213.612 & 0853.682 \\
\hline 54 & 13 & 7.740 & 5 & 0 & 0 & 13 & $16 / 06 / 2013$ & 4212.681 & 0854.428 \\
\hline 55 & 14 & 2.200 & 2 & 0 & 0 & 14 & 25/06/2013 & 4212.681 & 0854.428 \\
\hline 56 & 13 & 1.100 & 1 & 0 & 0 & 14 & 25/06/2013 & 42.13.162 & 08.53 .457 \\
\hline 57 & 14 & 0.492 & 1 & 0 & 0 & 14 & $25 / 06 / 2013$ & 42.13 .059 & 08.53 .454 \\
\hline 58 & 10 & 0.636 & 1 & 0 & 0 & 15 & 26/06/2013 & 42.13 .176 & 08.53 .529 \\
\hline 59 & 24 & 0.480 & 5 & 0 & 0 & 12 & 26/06/2013 & 42.13 .145 & 08.53 .740 \\
\hline 60 & 8 & 1.120 & 1 & 0 & 0 & 14 & $01 / 07 / 2013$ & 4213.079 & 0853.493 \\
\hline 61 & 10 & 1.664 & 5 & 0 & 0 & 13 & $31 / 07 / 2013$ & 4212.686 & 0854.871 \\
\hline 62 & 22 & 0.780 & 5 & 0 & 0 & 15 & 07/08/2013 & 4215.140 & 0854.547 \\
\hline 63 & 15 & 1.200 & 5 & 0 & 0 & 19 & 29/08/2013 & 4215.030 & 0855.140 \\
\hline 64 & 10 & 1.560 & 2 & 0 & 0 & 14 & 18/09/2013 & 4211.784 & 0853.506 \\
\hline 65 & 21 & 0.774 & 2 & 0 & 0 & 13 & 18/09/2013 & 4211.799 & 0853.271 \\
\hline 66 & 11 & 0.738 & 3 & 0 & 0 & 13 & 25/09/2013 & 4212.726 & 0853.970 \\
\hline 67 & 21 & 0.990 & 3 & 1 & 0 & 13 & 25/09/2013 & 4214.478 & 0853.807 \\
\hline 68 & 15 & 0.990 & 1 & 1 & 0 & 13 & 07/10/2013 & 4214.738 & 0854.214 \\
\hline 69 & 20 & 0.660 & 1 & 0 & 0 & 17 & 07/10/2013 & 4213.291 & 0853.744 \\
\hline 70 & 11 & 1.400 & 5 & 0 & 0 & 18 & $30 / 10 / 2013$ & 4213.300 & 0853.830 \\
\hline 71 & 18 & 0.900 & 1 & 0 & 0 & 16 & $30 / 10 / 2013$ & 4211.550 & 0853.187 \\
\hline 72 & 10 & 0.510 & 1 & 0 & 0 & 16 & $14 / 11 / 2013$ & 4212.700 & 0854.364 \\
\hline 73 & 20 & 1.180 & 1 & 0 & 0 & 15 & $14 / 11 / 2013$ & 4212.492 & 0854.815 \\
\hline 74 & 12 & 1.925 & 3 & 1 & 0 & 15 & $26 / 11 / 2013$ & 4212.773 & 0853.972 \\
\hline 75 & 21 & 1.760 & 5 & 0 & 0 & 12 & $26 / 11 / 2013$ & 4211.268 & 0854.505 \\
\hline 76 & 14 & 2.400 & 1 & 0 & 0 & 12 & 03/12/2013 & 4211.525 & 0853.789 \\
\hline 77 & 29 & 2.400 & 5 & 0 & 0 & 12 & 03/12/2013 & 4214.901 & 0854.296 \\
\hline 78 & 17 & 3.420 & 2 & 1 & 1 & 12 & 05/12/2013 & 4212.649 & 0854.669 \\
\hline 79 & 18 & 0.459 & 2 & 1 & 1 & 11 & $05 / 12 / 2013$ & 4212.902 & 0853.396 \\
\hline 80 & 12 & 1.760 & 5 & 0 & 0 & 12 & $21 / 01 / 2014$ & 4212.649 & 0854.482 \\
\hline 81 & 28 & 0.704 & 5 & 0 & 0 & 14 & $21 / 01 / 2014$ & $42.14,842$ & 08.54 .074 \\
\hline 82 & 12 & 1.360 & 2 & 0 & 1 & 14 & $19 / 02 / 2014$ & 42.13.688 & 08.53 .650 \\
\hline 83 & 25 & 0.160 & 2 & 0 & 1 & 12 & $19 / 02 / 2014$ & 42.13.677 & 08.53 .418 \\
\hline
\end{tabular}




\begin{tabular}{|c|c|c|c|c|c|c|c|c|c|}
\hline 84 & 8 & 0.152 & 2 & 1 & 1 & 12 & 07/03/2014 & 42.13 .548 & 08.53 .846 \\
\hline 85 & 18 & 0.420 & 3 & 1 & 0 & 12 & 07/03/2014 & 42.11 .579 & 08.56 .093 \\
\hline 86 & 10 & 0.492 & 2 & 1 & 1 & 12 & $12 / 03 / 2014$ & 42.11 .587 & 08.53 .306 \\
\hline 87 & 18 & 0.432 & 5 & 0 & 0 & 13 & $12 / 03 / 2014$ & 42.11 .371 & 08.53 .343 \\
\hline 88 & 11 & 0.360 & 2 & 0 & 1 & 13 & $20 / 03 / 2014$ & $42.11,657$ & 08.53 .375 \\
\hline 89 & 20 & 0.480 & 5 & 0 & 0 & 13 & $20 / 03 / 2014$ & 42.14.396 & 08.53 .597 \\
\hline 90 & 11 & 0.936 & 3 & 1 & 1 & 13 & $10 / 04 / 2014$ & 42.12 .732 & 08.54 .275 \\
\hline 91 & 22 & 1.575 & 5 & 0 & 0 & 14 & $10 / 04 / 2014$ & 42.14 .477 & 08.53 .637 \\
\hline 92 & 14 & 3.675 & 5 & 0 & 0 & 13 & $16 / 04 / 2014$ & 42.14 .487 & 08.54 .000 \\
\hline 93 & 22 & 3.540 & 5 & 0 & 0 & 14 & $16 / 04 / 2014$ & 42.14 .959 & 08.54 .428 \\
\hline 94 & 31 & 2.347 & 5 & 0 & 0 & 14 & $17 / 07 / 2014$ & 42.14 .901 & 08.54.296 \\
\hline
\end{tabular}

\section{References}

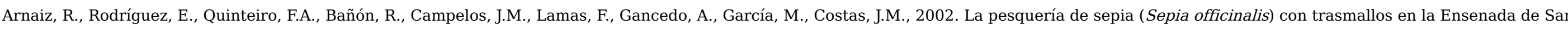

Simón (Ría de Vigo) 1999-2000. Los Recursos Marinos de Galicia, Serie Técnica 2. Xunta de Galicia, Santiago de Compostela, Spain, 57pp.

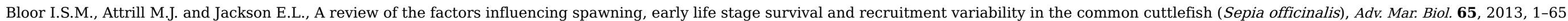

Boletzky S.V., Sepia officinalis, In: Boyle P.R., (Ed), Cephalopod Life Cycles, 1983, Academic Press London, 31-52.

CZFV (Consorcio Zona Franca Puerto de Vigo), Informe situación del sector pesquero, 2014, (consulted on 12.01.16) http://www.zonafrancavigo.com/red/index.php?

id=646\&option $=$ com content\&task=view\&Itemid=237\&idioma $=$ es.

Dunn M.R., Aspects of the stock dynamics and exploitation of cuttlefish Sepia officinalis (Linnaeus, 1758), in the English Channel, Fish. Res. 40, 1999, 277-293.

Forcada A.S., Evaluación de las Áreas Marinas Protegidas y su efecto en pesquerías artesanales del Mediterráneo Occidental, Ph. D. Thesis, 2007 , University of Alicante; Spain, 425.

Freire J. and García-Allut A., Socioeconomic and biological causes of management failures in European artisanal fisheries: the case of Galicia (NW Spain), Mar. Policy 24, 2000, 375-384.

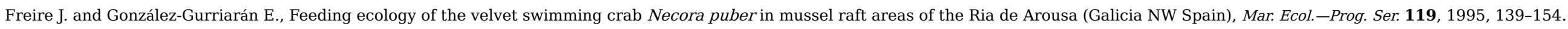

García, S.M., Zerbi, A., Aliaume, C., Do Chi, T., Laserre, G., 2003. The Ecosystem Approach to Fisheries. FAO Fish. Tech. Pap. No 443. FAO, Rome, 71pp.

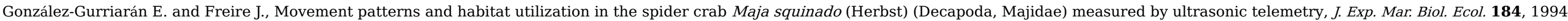
269-291.

Guerra A. and Castro B.G., On the life cycle of Sepia officinalis (Cephalopoda: Sepioidea) in the Ría de Vigo (NW Spain), Cah. Biol. Mar. 29, 1988, $395-405$.

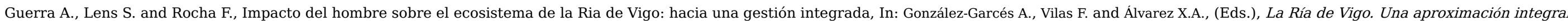
al ecosistema marino dela Ría de Vigo, 2008, Instituto de Estudios Vigueses; Vigo, 325-369.

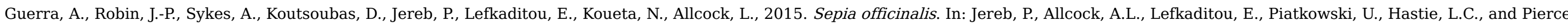
G.J. (eds) Cephalopod biology and fisheries in Europe: II. Species Accounts, pp 53-72. ICES Cooperative Research Report No. 325. 360pp. 


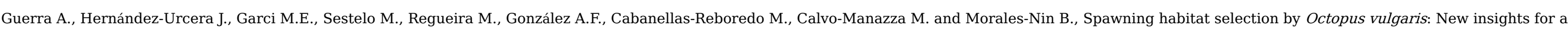
more effective management of this resource, Fish. Res. 167, 2015b, 313-322.

Guerra A., Ecology of Sepia officinalis, Vie Milieu 56, 2006, 97-107.

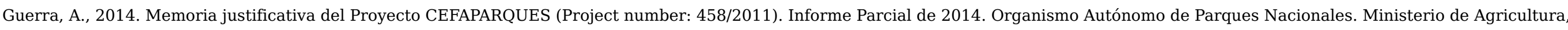
Alimentación y Medio Ambiente, Madrid (This rapport is available from the corresponding author.

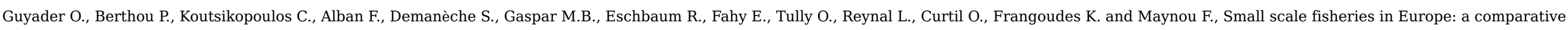
analysis based on a selection of case studies, Fish. Res. 140, 2013, 1-13.

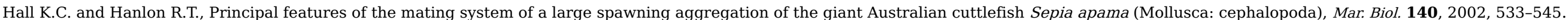

Hastie T. and Tibshirani R., Generalized Additive Models, 1990, Chapman and Hall London, 335.

Le Goff R.L. and Daguzan J., Growth and life cycles of the cuttlefish Sepia officinalis L. (Mollusca: Cephalopoda) in south brittany (France), Bull. Mar. Sci. 49, 1991, 341-348.

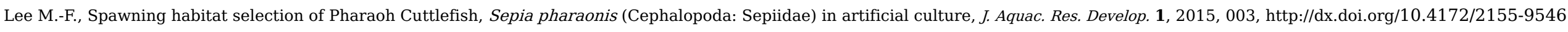

Mangold-Wirz K., Biologie des Céphalopodes benthiques et nectoniques de la Mer Catalane, Vie Milieu 13, 1963, 285.

McCullagh P. and Nelder J.A., Generalized linear models, Monographs on Statistics \& Applied Probability, second edition, 1989, Chapman \& Hall/CRC.

Moltschaniwskyj N.A. and Pecl G.T., Small-scale spatial and temporal patterns of egg production by the temperate loliginid squid Sepioteuthis australis, Mar. Biol. 142, 2003, 509-516.

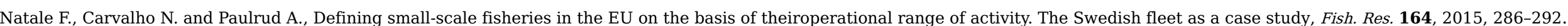

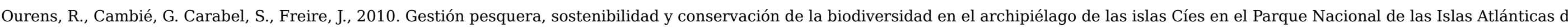
Galicia (GESCIES 030/SGTB/2007/1.4). Final Report. University of La Coruña, Spain, 209 pp.

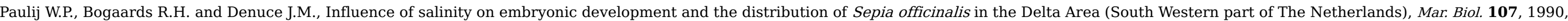
17-23.

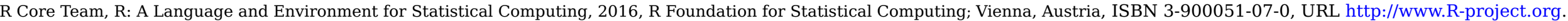

RAGG, Regional Autonomous Government of Galicia, 2015. DOGA, 16th July 2015, n. 133. Santiago de Compostela, Spain.

RAGG, Regional Autonomous Government of Galicia, Pesca de Galicia. Plataforma tecnolóxica da pesca, 2016, Available at: http://www.pescadegalicia.com.

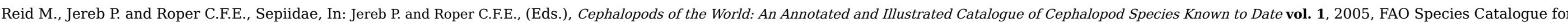
Fishery Purposes No. 4, FAO; Rome, 57-152.

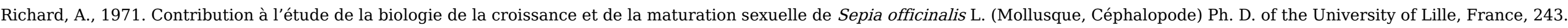
Roberts M.J. and Sauer W.H.H., Environment: the key to understanding the South African chokka squid (Loligo vulgaris reynaudii) life cycle and fishery?, Antarct. Sci. 6, $1994,249-258$.

Rocha F., Guerra A. and González A.F., A review of reproductive strategies in cephalopods, Biol. Rev. 76, 2001, 291-304.

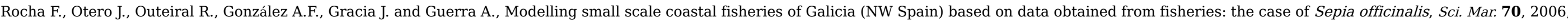
593-601.

Sampedro M.P. and González-Gurriarán E., Aggregating behaviour of the spider crab Maja squinado in shallow waters, J. Crustacean Biol. 24, 2004, 168-177. 


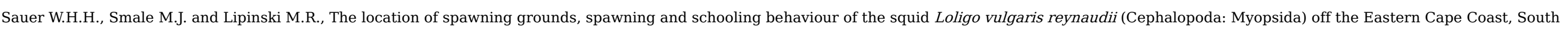
Africa, Mar. Biol. 114, 1992, 97-107.

Sestelo M., Villanueva N.M. and Roca-Pardiñas J., FWDselect: Selecting variables in regression models. R package version 2.1.0, 2015 http://cran.r-project.org/package=FWDselec.

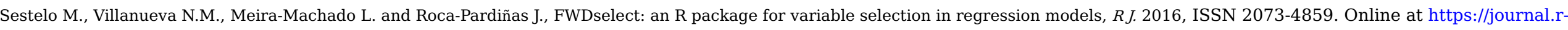

project.org/archive/accepted/sestelo-villanueva-meiramachado-etal.pdf.

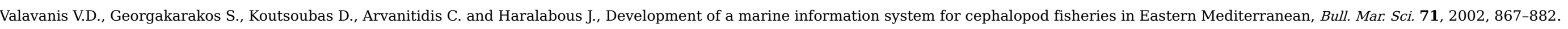

Valavanis V.D., Georgakarakos S., Kapantagakis A., Palialexis A. and Katara I., A GIS environmental modelling approach to essential fish habitat designation, Ecol. Model. 178, $2004,417-427$.

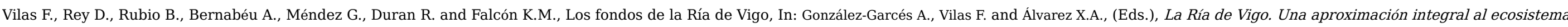
marino dela Ría de Vigo, 2008, Instituto de Estudios Vigueses; Vigo, 15-50.

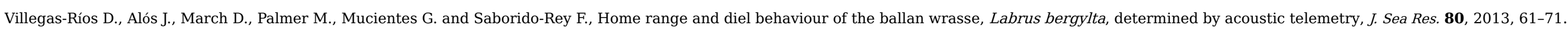

Wood S.N., Stable and efficient multiple smoothing parameter estimation for generalized additive models, J. Am. Statist. Assoc. 99, 2004, 673-686.

Wood S.N., Generalized Additive Models: An Introduction with R, 2006a, Chapman \& Hall/CRC Press; Boca Raton FL, 410.

Wood S.N., Low-rank scale invariant tensor product smooth for generalized additive mixed models, Biometrics 62, 2006b, 1025-1036.

\section{Queries and Answers}

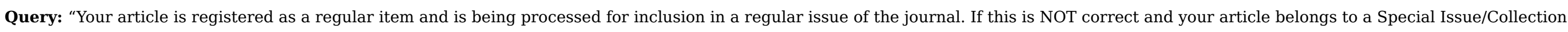
please contact i.gopinath@elsevier.com immediately prior to returning your corrections."

Answer: This is a regular paper.

Query: The author names have been tagged as given names and surnames (surnames are highlighted in teal color). Please confirm if they have been identified correctly

Answer: Please, delete the second e-mail address in the first author

All names identified correctly.

Query: Please check the presentation for all the affiliations, and correct if necessary.

Answer: Please remove Department of Natural Resources and Ecology and replace by ECOBIOMAR.

Query: Please check the e-mail address for corresponding author and correct if necessary.

Answer: Correct.

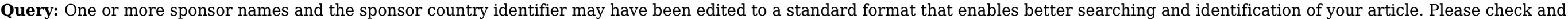
correct if necessary.

Answer: Correct.

Query: Please check the presentation for Ref. "McCullagh and Nelder, 1989", "Reid et al., 2005", "R Core Team, 2016" and correct if necessary.

Answer: Replace \& by and in McCullagh...

$\mathrm{R}$ Core Team is correct.

In Reid et al., italics could be removed. 
Query: Please provide the complete bibliography for Ref. "Richard, 1971".

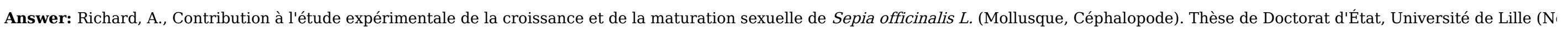
243), 1971, $264 \mathrm{pp}$.

Query: Please check the Figure 5 caption for correctness.

Answer: Fig 5. Number of Sepia officinalis egg masses per type of structure. Remove: where were fixed.

The H of Halydris should be written in italics.

Change boy for buoy.

Query: Please check the presentation for all the Tables and correct if necessary.

Answer: Correct.

Query: Please check the edits made in Table 2 and correct if necessary.

Answer: Margin for Table 2 out of page limit.

Sepia officinalis in italics.

Query: Please cite the "*" in Table 3.

Answer: Please, after Table 3 include Sepia officinalis.

Remove $(*)<0.1$.

May be data inside the table could be improved by centring them. 